\title{
The evolution of failure: explaining cancer as an evolutionary process
}

\author{
Christopher Lean ${ }^{1} \cdot$ Anya Plutynski ${ }^{2}$
}

Received: 22 November 2014/Accepted: 12 November 2015/Published online: 30 November 2015

(C) Springer Science+Business Media Dordrecht 2015

\begin{abstract}
One of the major developments in cancer research in recent years has been the construction of models that treat cancer as a cellular population subject to natural selection. We expand on this idea, drawing upon multilevel selection theory. Cancer is best understood in our view from a multilevel perspective, as both a byproduct of selection at other levels of organization, and as subject to selection (and drift) at several levels of organization. Cancer is a by-product in two senses. First, cancer cells co-opt signaling pathways that are otherwise adaptive at the organismic level. Second, cancer is also a by-product of features distinctive to the metazoan lineage: cellular plasticity and modularity. Applying the multilevel perspective in this way permits one to explain transitions in complexity and individuality in cancer progression. Our argument is a reply to Germain's (2012) scepticism towards the explanatory relevance of natural selection for cancer. The extent to which cancer fulfills the conditions for being a paradigmatic Darwinian population depends on the scale of analysis, and the details of the purported selective scenario. Taking a multilevel perspective clarifies some of the complexities surrounding how to best understand the relevance of evolutionary thinking in cancer progression.
\end{abstract}

Keywords Cancer · Multilevel selection - Darwinian populations · Tumors · By-product of selection

“Through failure we understand design.” Frank, 2007.

Christopher Lean

christopher.hunter.lean@gmail.com

1 School of Philosophy, Australian National University, Canberra, Australia

2 Department of Philosophy, Washington University in St. Louis, St. Louis, MO, USA 


\section{Cancer as an evolutionary process}

At first glance, cancer seems the epitome of disorganized growth, rather than adaptive evolutionary change. Indeed, cancer is often defined as a failure of the otherwise functional regulation of cell birth and death. This view would seem to be fundamentally at odds with treating cancer progression as a process of natural selection. However, a trait that is dysfunctional at one temporal or spatial scale of analysis may well constitute "efficient functioning" at another (Hausman 2012). Indeed, this should not surprise us, given that multicellular organisms are (more or less) hierarchically organized, with functional parts within parts, and subsystems within systems. There is an active research program underway that takes this insight as foundational; these researchers treat cancer progression as a process of natural selection on cells and cell lineages (Michor et al. 2004; Merlo et al. 2006; Anderson et al. 2006; Pepper et al. 2007; Greaves and Maley 2012). The idea that cancer progression may be viewed as an evolutionary process is not new: Gause (1966) compared a population of cancer cells to an evolving population of microbiota. Nowell (1976) and Cairns $(1975,1978)$ similarly argued that 'cancer can be viewed as the operation of Darwinian selection among competing populations of dividing cells' (Cairns 1978, 151). ${ }^{1}$ What started as a minority view has now become an active area of research, drawing upon mathematical modeling, computer simulation, experimental evolution in model systems, and even the generation of phylogenies, or branching evolution in cancer progression and metastasis, using high throughput sequencing of tumors and metastatic lesions from deceased patients (Gerlinger et al. 2012; Navin et al. 2011; Yachida et al. 2010).

Philosophers of biology have begun to take notice of this research. Peter Godfrey-Smith in 'Darwinian Populations and Natural Selection" refers to cancer progression as "re-Darwinization": a "decoupling" of cellular fitness from that of the organism (Godfrey-Smith 2009). The idea of cancer being more or less "decoupled" in its fitness from its host is a useful theoretical starting point, as it captures the two extremes of cancer's relationship to the body. Cancer cells are relatively autonomous as compared to other cells in the body, but autonomy is a matter of degree. In some rare cases, e.g., infectious canine cancers, cancer can become as autonomous from the host body as any virus is from its host. In the case of Tasmanian devils, and some Canines, there are truly transmissible cancers: Canine Transmissible Venereal Tumor CTVT and Devil Facial Tumor Disease (DFTD). However, most cancer cells are not autonomous in this strong sense: most cancer cells (in vivo) cannot transmit between hosts, or grow in culture. Indeed, it took decades to identify a cancer cell line (HELA) that could grow indefinitely in culture. What determines the degree of "autonomy" and fitness of cells and collectives is not only on cell-intrinsic features, but also local conditions, such as signals from neighboring cells, as well as structural features of the tissue microenvironment (Bissell and Hines 2011). In vivo, cancer cells' degree of autonomy changes over time, dependent upon these local conditions, as well as the stage of advancement of a cancer. While cancer cells are in competition, ultimately,

\footnotetext{
${ }^{1}$ For a history of evolutionary perspectives on cancer, see, Morange (2012).
} 
their survival and reproductive success also depends upon cooperative interactions with the tumor stroma, and the cooption of organismic adaptations. Below, we argue that cancer progression can display multilevel selection dynamics.

Recently, Germain (this journal 2012) has argued that the population of cells in a cancer is at best a "minimal" case of a Darwinian population. On his view, cancer does not acquire sufficiently complex adaptations to count as paradigmatically Darwinian, by the lights of Godfrey-Smiths' account of Darwinian populations (2009). We agree to an extent, but argue that Germain's focus is too narrowly on the individual cell. We argue that once one takes a multi-level perspective on cancer, cancer is meaningfully described as both process and byproduct of selection. That is, cancer progression involves cooption of adaptations at distinct levels of organization in the biological hierarchy. We argue that this multilevel perspective can shed light on both the dynamics of cancer progression, and differential vulnerability to cancer within and between species and higher clades. Indeed, taking an evolutionary perspective may assist in cancer treatment, such as demonstrating when and why it is better to use combinations of drugs to treat cancer (Komarova and Wodarz 2005). Our argument has two parts. In the section titled "Multi-level selection", we briefly explain and describe the multilevel perspective. In the section titled "Applying the models", we apply this perspective to cancer itself. In the section titled "Adaptation and levels", we explain how our view departs from Germain.

\section{Multi-level selection}

There is an extensive cannon of interdisciplinary work on multilevel selection (Lewontin 1970; Wilson 1975; Damuth and Heisler 1988; Michod 1997; Sober and Wilson 1999; Okasha 2005, 2006; Godfrey-Smith 2009). To understand tumor progression, we will draw on a few key ideas in this literature. First, multilevel selection theory starts with the relatively uncontroversial observation that populations of entities are nested within one another in the biological hierarchy: populations of genes are nested within cells, which are nested within organisms, and organisms are nested within populations, which are nested within species and so on to whole lineages. Provided that these entities vary, the variation is heritable, and makes a difference to relative fitness of variable entities, selection can act at more than one level in this hierarchy. For, in principle, any population of entities with heritable variation in fitness may evolve by natural selection (Lewontin 1970). In a "multilevel" selection situation, selection is acting on more than one level simultaneously. Selection at one level may increase or decrease the frequency of traits in a population, which in turn, may affect what is available to selection at a lower or higher level of analysis.

In multilevel selection, it can become difficult to discriminate which outcomes are due to selection at a "lower," and which at "higher" level of analysis. Damuth and Haisler introduced a useful distinction between multilevel selection 1 (MLS1) and multilevel selection 2 (MLS2) to assist in discriminating between different scenarios. As they put it, "of interest in the former case are the effects of group 
membership on individual fitnesses, and in the latter the tendencies for the groups themselves to go extinct or to found new groups (i.e., group fitnesses)" (Damuth and Heisler 1988, p. 407). That is, in MLS1, fitnesses are properties of individuals, and character values are attributed to individuals. But, group membership can have effects on individual fitness. When this happens, one has a case of MLS1. Because different groups have different mixes of individuals, and some mixes are more successful than others (i.e., the individuals in some groups better survive and reproduce than the same individuals in a different group with different proportions of variants), we may describe outcomes of such group effects as a product of "group selection" in the sense of MLS1. The outcome in such cases is simply changing proportions of different kinds of individuals in the whole population (the metapopulation). Damuth and Heisler explain that some have wished to call MLS1 "group selection" because group level properties (such as the relative proportion of altruists in a group) determine the relative success of these individuals.

But there is another sense of "group" selection that is often confused with this first sense: what Damuth and Heisler prefer to call multilevel selection 2, (MLS2). Here "group selection" refers to change in the frequencies of different kinds of groups, in light of "group level" properties. This sense of group selection is more controversial, not least because groups are often diffuse in space and time, and their properties also are difficult to define and measure. However, in MLS2, character values are attributed to groups (including both aggregate and global characters), and it is these group level characters that make a difference to group success at propagation, or the seeding of more groups. Groups are more "fit" if and only if they propagate more groups. To be clear, it is not a necessary condition of MLS2, according to Damuth and Heisler, that group characters be "emergent," in the strong sense that there is no way to explain the properties of the group in terms of the properties of the individuals. Average height or extent of genetic heterogeneity in a population is, after all, a group level trait, but it can be explained in terms of the properties of individuals that make up the group. All that MLS2 requires is that these group level properties are what cause group success, (measured in this case by the seeding or propagation of new groups, in the way that colonists succeed at colonizing new islands) (Damuth and Heisler 1988). As a vivid but simple example, consider two groups of organisms: one that is genetically uniform, and one with a large amount of genetic variation. In the latter group, a greater extent of genetic variation may better enable such groups to propagate and diversify in new environmental niches. Insofar as this group level trait (extent of genetic heterogeneity) was a cause of relative group success at propagation, this would count as an instance of MLS2.

Multilevel selection theory has been used to explain the emergence of new levels of organization in the biological hierarchy, sometimes called the "major transitions in evolution." (Okasha 2005, 2006). The argument is as follows: the evolution of multicellularity required the emergence of cooperative interactions between populations of cells. Or, there were intermediate stages between functionally integrated individuals, and mere collectives. This may have involved the cooption of traits initially adaptive at the individual level in service of collective benefit. 
Quorum sensing in bacteria may be a vivid example. Quorum sensing is a method by which bacteria detect local cell density by diffusion and accumulation of signaling molecules in the surrounding environment. At certain densities, the population as a whole can engage in a coordinated response, e.g., the production of bioluminescence, population mobility, biofilm maturation, and virulence (Williams et al. 2007). That is, quorum sensing regulates production of "public goods," or products manufactured by an individual that can then be utilized by the individual or its neighbors. The capacity to quorum sense likely evolved from "self-signaling" systems that were adaptive at the individual level. (Redfield 2002; Williams et al. 2007). There are many such cases of proto-organismic collectives in nature. Or, as Queller and Strassman put it, there are:

... levels of organism, and each level was attained by merging formerly separate individuals at a lower level.... Multi-cellular individuals are cooperative groups of cells, eukaryotic cells are cooperative assemblages of multiple prokaryotic lineages and prokaryotic cells must have emerged by assembly of formerly independent replicators. These major transitions in evolution construct new levels of organism out of separate individuals (Queller and Strassman 2009, p. 3143).

How do these transitions come about? Multilevel selection theory figures in one prominent account: During the major transitions in evolution, e.g., the transition to multicellularity, the fitness of the collective must override the fitness interests of the parts. That is, collectives or groups must become more successful as collectives; i.e., they have to outcompete other collectives in reproduction, or, in propagation of further collectives. Cooperation and functional integration of parts can play a role in the evolution of such a collective. Cooption, or cross-level byproducts, might also play a role in the emergence of functional integration. As in the case of quorum sensing, traits that originally may have enhanced individual fitness can be coopted in service of collective fitness, or the relative success of groups at propagation. Some have argued that what might begin as a "group selection" process in the sense of MLS1 (individuals benefitting due to group membership) could evolve into a circumstance where we have a case of MLS2 (groups being more or less successful at propagation, due to group level properties) (Michod and Herron 2006; Okasha 2006). That is, the major transitions in evolution may have involved successive changes in population structure and organization. Individuals gradually organized into collectives, benefited from their membership in some collectives, and eventually, came to succeed or fail as collectives (Okasha 2005). If particle fitness is improved by being part of a particular collective, then we can speak of the particles, which gain mutual advantage within the collective, as having "aligned" fitness. Aligned fitness allows for the emergence of complex integration and interaction within the collective. Integration and particle interaction can lead to collectives that are functionally organized, so that the collective fitness of the group is distinct from that of its members. In this way, collectives with division of labor are, as Queller and Strassman argue, akin to organisms, to a greater or lesser degree. We will argue that something approaching this process may occur in advanced tumors. 
To summarize: in principle, selection can act within a collective-among the parts-and, between collectives. These processes could be going on simultaneously, or sequentially. Damuth and Heisler (1988) describe two senses of multi-level selection, "MLS $2 \mathrm{v}$. MLS 2" to highlight the fact that "group selection" may refer to different things, and that the collective fitness of a group is not necessarily simply a matter of averaging over particle fitnesses (See Table 1 for summary). This is the central point of critics of genic selection (see, e.g., Sober and Wilson 1999): simply averaging fitness of different genotypes that live in distinct groups may result in a loss of important causal information. As Wilson demonstrated (1979), group membership can make a difference to relative fitness; for instance, altruism can evolve in "trait groups"-where population structure is not freely mixing, but where similar individuals tend to find themselves together. Cooperation is less likely to evolve in a freely mixing population, where "cheaters" or "free riders" can arise and spread. In trait groups, the fitness of individuals is largely contingent on the make up of their group. Nor is the fitness of a group simply the average fitness of its members: the two can come apart and pull in different directions. Some groups may propagate more successfully than other groups, even if the average fitness of the group's members is low (for discussion, see Sterelny et al. 2014).

\section{Applying the models}

In order to see why these models may be applied to cancer, there are some basic facts about cancer initiation and progression that need to be made clear. Many people think that cancer is a rare and unusual event; this is a natural assumption to make, since we are made aware of cancer on the occasion of diagnosis of invasive disease. But, invasive cancer-that is, what clinicians define as cancer-is the endpoint of a long process; most early stages of this process fail to advance to an invasive cancer (Sakr et al. 1993; Mori et al. 2002; Martincorena et al. 2015). Over the course of the lifetime of the average individual, there are many thousands of cell

Table 1 Multilevel selection

\begin{tabular}{|c|c|c|}
\hline $\begin{array}{l}\text { Multilevel selection } \\
\text { (Damuth and Heisler } \\
\text { 1988) }\end{array}$ & MLS1 & MLS2 \\
\hline $\begin{array}{l}\text { "Group selection" } \\
\text { refers to }\end{array}$ & $\begin{array}{l}\text { Change in frequency of } \\
\text { individuals in light of group } \\
\text { membership }\end{array}$ & $\begin{array}{l}\text { Change in frequencies of different kinds of } \\
\text { groups }\end{array}$ \\
\hline $\begin{array}{l}\text { Fitnesses are } \\
\text { properties of }\end{array}$ & Individuals & Groups \\
\hline $\begin{array}{l}\text { Characters are } \\
\text { values attributed to }\end{array}$ & Individuals (e.g. altruism) & $\begin{array}{l}\text { Groups (e.g., group mean, population } \\
\text { density, proportion of different phenotypes) }\end{array}$ \\
\hline $\begin{array}{l}\text { Appropriate for } \\
\text { investigation of }\end{array}$ & $\begin{array}{l}\text { Evolution of characters of } \\
\text { individuals likely affected by } \\
\text { group membership }\end{array}$ & $\begin{array}{l}\text { Changing proportions of different types of } \\
\text { groups; different propensities to go extinct } \\
\text { or to found new groups }\end{array}$ \\
\hline
\end{tabular}


divisions, and randomly distributed somatic mutations occur and accumulate in normal cells. Only a very small proportion of these cells and their descendants succeed at advancing to cancer. What molecular biologists and modelers of cancer's evolutionary dynamics are hoping to predict and explain is which ones will succeed at advancing to an invasive cancer, and why. Some investigators focus on the particular features of the cells themselves; some focus on features of whole populations of such cells; some focus on hierarchical or spatial relations between cells; some focus on interactions between cancer cells and their microenvironment. Whether a cancer progresses is likely due to all of the above. Our view is that by taking a multilevel evolutionary perspective we can integrate a variety of levels of analysis, or show how they are mutually constraining and mutually informative. That is, in our view, there are several different targets or levels of selection at play in cancer initiation and progression, some more salient at different stages of progression.

Identifying a level of selection involves identifying a population of like entities, which have reproduction, and differential fitness due to heritable features of such entities (Godfrey-Smith 2009). According to the multilevel selection view, there are several distinct units or targets of selection potentially of relevance in cancer progression. These are not mutually exclusive options, but may be co-occurring:

1. Individual selection, mutation, and drift, acting among single cells, whether "pre-cancer" cells, cancer cells located in a tumor, or metastatic populations of cells. Individual cells may be more or less fit in the sense of overcoming the variety of constraints in normal tissue that ordinarily prevent disorderly cell growth, acquiring resources in the local tissue microenvironment, or adapting to novel tissue microenvironments (in the case of tumor metastases).

2. "Group" selection in the sense of MLS1 or MLS2: Some individuals may survive and reproduce more successfully than others, because of membership in a group (MLS1). Alternatively relative fitness may be ascribed to groups as a whole (MLS2). Fitness in the latter case is assessed by the ability of the tumor to produce new tumors, due to properties of the group-these may be either aggregate properties (e.g., population size, extent of tumor heterogeneity), or interactive properties (functional interactions between cells or lineages in a tumor).

We may think of the process of cancer progression as proceeding through a series of stages, where individual selection is more salient in the beginning, and gradually, MLS1 and MLS2 become salient (See Fig. 1). This is, arguably, not unlike the process some have hypothesized was at work in the emergence of multicellularity, as discussed above (see, e.g., Okasha 2006). Most models of the evolutionary dynamics of cancer are simple individual level selection models (Nowak 2006; Frank and Nowak 2004; Michor et al. 2004), where the fitness advantage is attributed to a single cell, due to a gain of function or loss of function, attributable to a single mutation. Such mutations can yield advantages such as promoting angiogenesis, blocking tumor suppression, or inhibitors of growth (halting or blocking apoptosis, or cell death), or promoting cell division (e.g., gain of function 


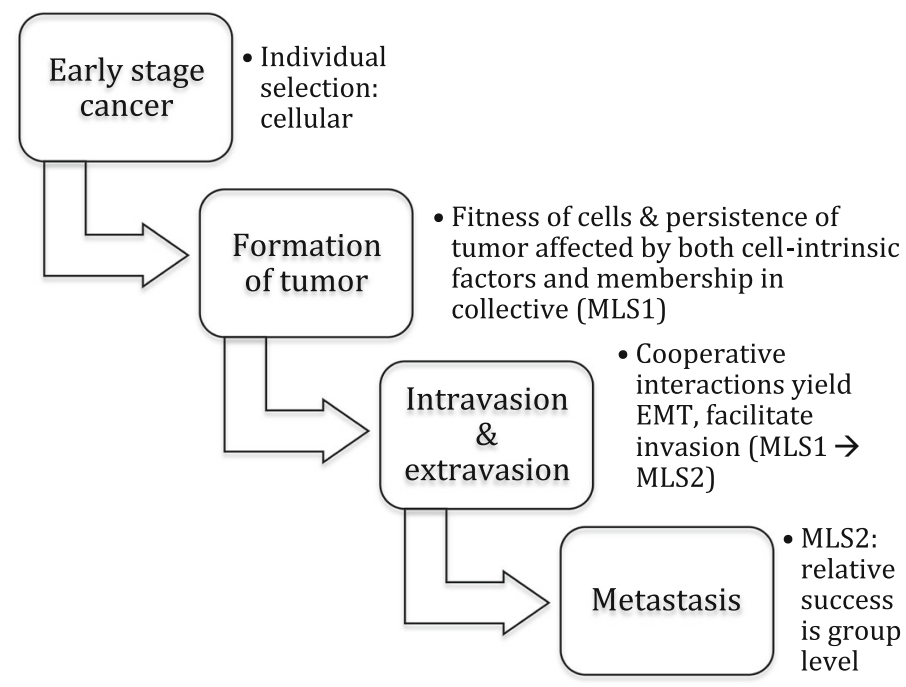

Fig. 1 Transitions in levels of selection as cancer progresses

mutations). Growth in any environment will be limited by space and availability of nutrients; among incipient cancer cells, some cells will be better able to compete for these resources, or escape mechanisms of homeostatic control. Such evolutionary dynamics are taken to feature centrally in early stages of the development of a "proto-" cancer cell lineage. Models of such circumstances look not unlike competitive exclusion models in ecology (See Wodarz and Komarova 2014 for a review).

There could also be selection operating at the level of cell colonies, however. Komarova and Wodarz $(2004,2014)$ argue that there is an optimal "strategy" for chromosomal instability (CIN) or rate of loss of heterozygosity in cancer. Cancer cells characteristically have various chromosomal abnormalities-duplications, losses, and inversions of large parts of the genome complement of a cell. One might think chromosomal instability is uniformly bad, but chromosomal instability in cancer is a "two edged sword": too much instability can lead to destructive genomic changes, such as the loss or destruction of whole chromosomes. In such cases, cells simply cannot replicate and will die off. But, if timed correctly, CIN can inactivate tumor suppressor genes, for instance, and thus promote clonal expansion. Some lineages will thus be more successful than others in progression to metastasis due to the specific types of traits of cells, acquired at specific times or stages in the progression of the disease. But, some collectives, or colonies of proto-cancer cells, may more quickly reach a cancerous state, insofar as they have the optimal "strategy" - or, the optimal growth rate given a certain degree of instability; too much instability is detrimental because it increases the death rate. ${ }^{2}$ Too little slows down progress because the rate of onset of cancerous mutations is low. Of course,

\footnotetext{
${ }^{2}$ A reviewer asks whether the lineage of cells in a clonal population is a "population." We grant that it is an atypical population, in that the population is all descended from a clone. But, if clonal populations of
} 
this is an idealized model, and populations of pre-cancerous cells are not a simple series of clonal expansions, acquiring a specific mutation at each stage, and not "adjusting" their rate "strategically." But, Komarova and Wodarz's point is a theoretical one: Early acquisition of chromosomal instability carries an advantage, which, they argue, falls off over time, as too much genetic instability overall reduces fitness. Lineages with the optimal strategy are the ones that "succeed" in progressing to cancer. Evidence that there are many "potential" cancers comes from samples of otherwise healthy tissue containing cancer mutations (Martincorena et al. 2015).

Moreover, population architecture could well predict the evolution of faster or slower progression to a cancer phenotype; that is, where most of the tumor is maintained by a small number of tumor stem cells, the pattern of stem cell division can affect rate of escape from feedback regulation, and thus also the acceleration of onset invasive cancer. Arguably, such a case would count as an instance of both simple individual selection, and MLS2. For, the time to the evolution of escape from feedback regulation depends upon effective population size, which is a group level property (MLS2). The "cancer stem cell" hypothesis holds that some cells in a tumor have "stem-like" properties. Such cells are constantly renewing and thus more likely to both seed new tumors and perhaps also survive chemotherapy (Jordan et al. 2006). While the cancer stem cell hypothesis is somewhat controversial (see, e.g., Gupta et al. 2009; Nguyen et al. 2010), it is certainly true that some cells in a cancer appear to be better able to seed new tumors.

Critics of the Darwinian picture have argued that models of cancer evolution treat cancer as a simple clonal expansion process, where the population of cells is relatively homogeneous (see, e.g., Weinberg 2014, pp. 458-468). We now know that tumors are heterogeneous populations. However, there is no reason in principle why population structure in a tumor cannot be incorporated into an evolutionary model (indeed, it's been done already, see, e.g., Wodarz and Komarova 2014). One simply reduces the effective population size of a tumor, where the stem cells are the only constantly renewing cells. Moreover, one could view a tumor as several co-evolving lineages, each competing for space and resources. Such co-evolving subpopulations-or the coevolution of metapopulations-is common in nature, and has been studied in a variety of species where lineages are subdivided into local niches and so may evolve either local adaptations, or engage in competition or co-evolutionary dynamics. Weinberg himself considers whether "distinct subpopulations of cells within a tumor act symbiotically, each supporting the growth and survival of the other, rather than directly competing" (p. 466). Such coevolving lineages would count as a case of a higher level of selection, either in the sense of MLS 1 or 2; e.g., cells can benefit from membership in a tumor with a neighboring cells or cell lineages that produce acidic byproducts that enable metastasis (Gatenby 2006); or, heterogeneous tumors may be more successful at acquiring resources, resisting attacks from the immune system, or seeding metastases, which effectively counts as an instance of MLS2. Such different scenarios simply require that we adopt a multilevel perspective.

Footnote 2 continued

bacteria such as Lenski's E. coli are populations, we don't see why clonal populations in a tumor would not be. 
That is, MLS2 becomes more relevant as we consider malignancy, or invasion and metastasis. This process requires that the carcinoma grow to a stage where it can acquire a novel phenotype (motility), and thus reproduce: metastasis is the production of other tumors throughout the body. But this process is not simply the release of a single cell into the blood or lymphatic system. "Seeding" of metastases is an extended process that involves a complex series of steps: the breaching of the basement membrane, intravasion, the invasion or dissemination of metastatic cells, and the colonization of distant organ sites. This process requires the cooperation of tumor cells and stroma; the cooption of the very same heterotypic interactions that are ordinarily used in service of wound healing (see, e.g., Weinberg 2014, pp. 587-607). Most attempts at seeding, or most incipient metastases fail; the disseminated cells more often than not are destroyed in circulation. The ability of micrometastasis to colonize remote sites is not simply a given, in other words, but requires many attempts, akin to the colonization of remote islands by Darwin's finches. Some metastatic populations are more effective at colonization than others, and once a metastasis is established, these colonies, in turn, can be more or less successful at disseminating secondary metastases. The secondary wave of metastasis is sometimes called a "metastatic shower" because they send off many "showers" of potential colonists (cf. Weinberg 2014, p. 654).

The success of metastases is not due exclusively to properties of metastatic cells. Metastasis involves the cooperation of the stromal tissue; activation of the EMT (the epithelial mesenchymal transition) in carcinoma requires signals from the tissue microenvironment; and the tumor both recruits stromal cells (macrophages, mast cells, fibroblasts) and coopts signals ordinarily associated with wound healing to break down surrounding tissue and metastasize. That is, the entire process of metastasis is not achieved by single cells in isolation, but the activity of both the tumor and tumor-associated cells in stimulating angiogenesis, breaking down surrounding tissue, and enabling intravasation. In other words, while it is single cells that eventually establish metastases, the process of metastasis is enabled by and indeed functionally requires the interaction of many cells in the tumor and surrounding tissue. We might think about metastasis as rather like " $r$ " selection rather than " $\mathrm{k}$ " selection; given that the environment is unstable, and most metastases fail, tumors more successful at sending out large populations of multiple metastases are more likely to be successful. ${ }^{3}$ More heterogeneous primary tumors in the sense of both more genetically diverse, and epigenetically plastic and modular - may be able to generate more and more successful metastases (Weigelt et al. 2003; Klein et al. 2002).

Evolutionary dynamics also apply outside the primary tumor. Metastatic populations continue to evolve in their new environments, and may be more or less successful at colonizing, and generating successful secondary colonists (or, secondary metastases), in light of the properties inherited from the primary tumor (Psaila and Lyden 2009). The various properties of the primary tumor can be

\footnotetext{
3 A ' $r$ ' selection population is one with a high growth rate, seeding many individuals. ' $\mathrm{R}$ ' selection is often associated with populations which gain from dispersing over hostile environments. In contrast ' $\mathrm{k}$ ' selection population have low growth rates due to the population living in an environment near carrying capacity. See Macarthur and Wilson (1967).
} 
maintained in secondary tumors. Evidence for fidelity in reproduction include micro-assays of breast cancer showing that gene expression is maintained across metastatic sites (Weigelt et al. 2003). There is in fact evidence that the ability to colonize itself evolves (see Klein et al. 2002). Differential success is due in part to group level properties of the primary tumor, namely, genetic heterogeneity and functional diversity. Different cell lines in a tumor acquire differential capacities that combined lead to more successful reproduction of secondary metastases at different sites. All in all, provided that the fitness-increasing properties of the group are reproduced in the novel site, we have a case of MLS2. If multiple metastases arise from the primary tumor, and the tumor is heterogeneous, then it is possible for the heterogeneity of the primary tumor to be found likewise in the metastatic sites. Indeed, we urge further work on this empirically, but we believe that the evidence already suggests that looking outside of cell-intrinsic properties to understand the evolutionary dynamics of metastasis is essential. ${ }^{4}$

Thus, depending on the level of analysis and the empirical details of any particular case, cancer progression could be viewed as an individual level selection process, or a group level selection process, in the sense of MLS1 or MLS2. That is, a cancer cell could be competing with, or co-evolving with other cell lineages, or incipient cancers or proto-cancer cell populations might be competing with other proto-cancers for space, and resources. Or, differential reproduction via differential success at invasion and metastases could be due to complex properties of the tumor as a whole, including functional differentiation within a tumor, and cooption of organismic adaptations in service of invasion and metastasis. That is, if invasion and metastasis requires the acquisition of several capacities, such as the capacity to coopt certain signaling systems in the microenvironment, different cell lineages within a tumor might acquire these different capacities over time, and together cooperatively produce the cancer phenotype. Komrova and Wodarz make the point quite simply:

Assume that tumor progression requires the presence of three gene products, call them A, B and C. A cell can evolve to accumulate all three mutations, thus

\footnotetext{
4 A reviewer comments: "There is no denying that the success of metastasis depends on many evolved features and interactions (including cells other than the colonizing cells) and, as the author points out, that features such as heterogeneity are tumor-level property... However, in order to show that tumours have complex adaptations, it is insufficient to show that tumours have developed complex traits, or that these promote progression, and one must also show that these traits were selected at this level, rather than for the benefit of single cells." This is an excellent comment and central concern worth addressing. In our view, a trait that promotes relative survival and reproductive success of an entity at some level need not be (initially) selected for survival and reproductive success at that particular level. As long as it (currently) promotes survival and reproductive success of entities at that level, the trait is selected for at that level. We believe that we have shown this to be the case, and, the following references provide further elaboration of examples (Gatenby et al. 2007; Gavert and Ben-Ze'ev 2010; Egeblad et al. 2010; Mueller and Fuesnig 2004). Ruling out selection on a given level by arguing that a trait was not initially selected for to benefit that level would rule out all selection on all coopted traits, which would (potentially) rule out a good deal of selection even at the level of individual cells or organisms. See, e.g., Lloyd (2012) for discussion.
} 
achieving the malignant phenotype. On the other hand, the three gene products can arise independently in different cells, and they can share the gene product as a public good. In this setting, the malignant phenotype arises as an emergent property from a collection of cooperating cells rather than an individual cell (Wodarz and Komarova 2014, p. 460-1).

Put simply, on the MLS2 view, collectives are more or less successful in light of group level properties: heterogeneity, or division of labor in the process of invasion and metastasis. In the latter case, the collective of cell lineages in the tumor in some sense has "aligned" fitnesses. Reproductive "success" in such cases is measured in terms of invasion and propagation of metastases (recall: most incipient cancers fail to progress); or, metastases could be more or less successful at generating secondary metastases. In MLS2, we are interested in the way group level traits affect group level fitness. Thus, cell lineages (including cancer and non-cancer stromal cells) might interact as a collective (both with other cancer cells and with the tumor stroma) thus increasing the collective's ability to survive and reproduce, relative to other collectives (which might include other precancerous lesions, or other metastases, at seeding further "secondary" metastases). If better functional integration in a tumor results in more successful metastasis, then the collective fitness of the whole is distinct from the aggregative fitness of the individual cells. There is now ample evidence that tumors are complex heterogeneous populations of cells, and that signaling between these cells plays an essential role in tumor progression (Egeblad et al. 2010).

Cancer cells also recruit neighboring cells in order to participate in tumor development, both a structural support, and in building a blood supply (Mueller and Fuesnig 2004). Arguably, this is a form of niche construction in which the cancer cells and normally functioning cellular populations both evolve and co-evolve with their surrounding environments (Odling-Smee et al. 2003). That is, cancer progression requires the cooperation of the tissue microenvironment; the behavior of a tumor is not due exclusively to the behavior of cancer (or, neoplastic) cells alone. Carcinoma cells release growth factors that recruit macrophages, neutrophils, and lymphocytes, which in turn orchestrate an inflammatory response that involves the release of signals that stimulate the proliferation of epithelial cells and the process of angiogenesis in the tumor stroma. Epithelial cells release PDGF, for which fibroblasts \& myofibroblasts have receptors; these in turn release IGF-1, which benefits growth and survival of nearby cancer cells. Stromal and epithelial cells collaborate in construction of the extracellular matrix (ECM). Stromal cells are recruited by tumor cells to aid in construction of tumor colonies. Metastatic cells can spread throughout the body because they can acquire a "disguise" of platelets that enable them to evade detection (Egeblad et al. 2010). In all these cases, cancer cells are exploiting preexisting biological programs that are normally used by tissues for other purposes, in particular, wound healing. Tumor cells are activating a complex program that is already available to cancer cells, insofar as they evolved from healthy cells with the very same capacities. Wound healing uses a number of growth factors to increase permeability of the blood vessels near the wound, attract fibrogen to create blood clots, and attract fibroblasts, convert them into 
myofibroblast, which in turn release matrix metalloproteinases (MMPs) which play a role in building up the extracellular matrix, or remodeling the ECM, carving out spaces for new cells. Recruiting fibroblasts to the tumor allows the creation of a fibrin matrix, which serves as scaffolding in the initial formation of a tumor. It's important to see that this is not in tension with natural selection; selection and niche construction could be going on simultaneously. Those types more effective at shaping their environment to suit their ends will outcompete their peers.

The cooperation of the tumor cells and stromal cells is not simply a cooption of existing program of cells, however. The cancer cells and non-cancer cells may coevolve; that is, cancer-associated stromal cells become more adept over time at assisting in the survival of cancer cells within the tumor. Experimental evidence for the importance of the stromal tissue in tumor progression comes from a variety of sources, but we will discuss just one powerful case study. CAFs are cancerassociated fibroblasts, very similar to fibroblasts in normal cellular tissue, which play a role in building up scaffolding in injured tissue. An experiment to test the role of CAFs mixed normal human prostate cancer cells in combination with different kinds of fibroblastic cells, and then implanted them in host immunocompromised Nude mice. When cancer cells were mixed with CAFs, they grew tumors 500 times as large than when mixed with fibroblasts from normal prostate glands prior to injection into hosts (Olumi et al. 1999). Weinberg argues that the cumulative evidence of the role of CAFs in tumor formation suggests that "stromal cells in advanced carcinomas, having coexisted with epithelial cancer cells for many years, may change their genotype and acquire traits that genetically normal stromal cells cannot achieve. This suggests that stromal cells co-evolve with their neoplastic neighbors during long periods of tumor development." (Weinberg 603) In other words, a tumor (which is composed not only of cancer cells, but also of stromal tissue) is a complex tissue with co-evolving cell lineages, composed not only of cancer cells, but also non-cancer cells, which together cooperate in the progression of a tumor to metastasis. Indeed, Egeblad et al. (2010) claim that the highly integrative functional organization of some tumors suggests that they should be considered as akin to organs.

It's important to note, as Fisher pointed out, natural selection is not evolution (Fisher 1930). While there are multiple levels of selection in operation that potentially play an explanatory role in cancer, we also grant that there must be a role for drift. There is no doubt that the population size of cells in a tumor as a whole is sufficiently large for selection to overcome drift in the tumor as a whole; the volume of a cubic centimeter within a tumor cell mass contains about $10^{9}$ cells. However, tumors - to say the least-have a complicated population structure. It is quite likely that whether a cancer cell initially lives or dies may have to do as much with accidents of location as particular fitness-enhancing features intrinsic to the cell. The relative importance of selection versus drift will vary case by case; for instance, there are likely many isolated sublines of cancer cells within a tumor that may or may not survive, given lack of access to a blood supply, or architectural features of the tissue microenvironment, rather than features intrinsic to the cancer cells or populations of such cells (Crespi and Summers 2006). The threshold of .2 $\mathrm{mm}$ is the maximum required for oxygen to effectively diffuse between tissues; so, tumors will 
not grow if greater than $.2 \mathrm{~mm}$ distance from blood vessels. This mimics the way the fitness of normally functioning somatic cells is determined by their local cellular signaling environment rather than their own intrinsic features. Further, where a cancer is initiated may affect how slowly or quickly it progresses. Drift may well explain a good deal of variation we find in whether and why tumors progress to metastasis. And, if stem cell architecture affects tumor growth, such that only certain subpopulations are driving growth, drift will play a significant role.

The relevant grain of analyses and thus level of selection which explains any given phenomena of interest will be in part dictated by the questions we ask. If we are interested primarily in which cells come to predominate in a tumor, then a selection process within the population of cancer cells itself is a better target. However, if we are interested in using the body's own resources to fight cancer (e.g., via the development of immune therapies), perhaps we may be interested in viewing the tumor and stromal tissue as a whole (and its collective fitness enhancing properties) as a subject of analysis. Thus, there is a pragmatic dimension to the question: where is selection acting? But, there is also an empirical fact of the matter that constrains whether and to what extent selection (or, perhaps drift) is genuinely responsible for a given outcome of interest.

In sum, this multi-level perspective provides a representation of cancer progression that is both predictive and explanatory. The accumulation of mutations (and epigenetic changes) in neoplastic progression involve heritable variation in fitness of cancer cells and lineages, e.g., timing and acquisition of particular mutation types, chromosomal instability, hypoxia, acid resistance, transition to mesenchymal phenotype. Cancer progression involves several transitions in individuality and levels of selection, and, is a unique case of multi-level selection: it evolves at the level of populations of cells, competition among cell lineages, via cooption, a kind of cross-level exaptation, and as a group, or higher level "individual."

\section{Adaptation and levels}

Recently it has been claimed that somatic cell evolution by natural selection is not particularly relevant to understanding cancer (Germain 2012). Germain claims that while cancer meets the minimal requirements for natural selection, as described in Godfrey-Smith's Darwinian Populations, the main cases described as adaptations via a natural selection are not "complex" adaptations. Given that natural selection cannot explain the formation of such adaptations, he claims that it lacks explanatory power.

A central presupposition in Germain's argument is that he holds that for any feature to be an adaptation it must be the product of a gradual accumulation of iterative changes to the phenotypes. Each case Germain discusses is the product of a single large change. Senescence and apoptosis avoidance are products of mutations to TP53; immune evasion is gained through the loss of Human Leukocyte Antigen (HLA); and resistance to therapy is described as the loss of the expression of a targeting intermediate for the attachment of drugs or the activation molecular means 
for resisting drug uptake. In all these cases, Germain argues that the adaptation in question is due to a single mutation, not the product of cumulative change, and therefore not meaningfully described as complex adaptations.

Whether or not a trait is due to a single mutation of large effect or many mutations of small effect is irrelevant to whether it is in fact an adaptation. As Turner (1977) showed, mimicry in butterflies (a significant adaptive trait) can in some cases be due to major mutations with large effects on fitness, followed by mutations of small effect. But, there is a larger point to be considered here. Whether a trait is an adaptation, and for what, will depend on the grain of analysis or entity that the phenotype is predicated of. If selection only occurs in populations of cancer cells, and adaptations only to the single cell, affecting only its immediate descendants, then a Darwinian perspective would indeed not be very informative. However, if we "scale up" or "zoom out" on the population over a longer time scale, it becomes less implausible to see these adaptations as cumulative changes. If what we are interested in is the production of the cancerous phenotype as described by something similar to "The Hallmarks of Cancer" then a natural selection framework is very informative ${ }^{5}$. While some of the genes described by Germain are definitely important for a cancer cell's survival, they only can be treated as part of a "cumulative" adaptation insofar as collectively they contribute to a tumor's growth, persistence and metastasis.

Many of the cancer adaptations only appear to make sense at the tumor level, or at the level of collective fitness, where the collective includes the stromal tissue. There is evidence of cooperative coevolution between both cancer and stromal cells, each contributing to cycles of signals that promote growth, reshape the extracellular matrix, and up regulation of growth factors to produce angiogenesis, as well as promote invasion and metastasis. There is an incredible wealth of such positive feedback loops between carcinoma cells and what are called "Tumor-associated Macrophages," "Cancer-Associated Fibroblasts," and other cells in the reactive stroma. Acquisition of malignancy is not, in other words, a product of a series of mutations alone, but of the collaboration of both mutant alleles in cancer cell genomes and the signals that these cells send and receive from the tumor microenvironment (Karamysheva 2008). If we analyse a particular mutation of an individual cell that up regulates any of these growth factors we would not be able to say there was adaptation. However, the impact of multiple mutations can be cumulative and amplified by positive feedback, yielding, e.g., the angiogenic switch. That is, angiogenesis is product of the aggregation of multiple cell line mutations, and interaction between carcinoma and stroma. This is a stepwise accumulation of changes in the population that produces an adaptive phenotype for the tumor as a whole.

The key is to see this as a multilevel selective process, with selection operating at distinct levels simultaneously. There are enough features in common between the production of the cancerous phenotype and the production of other phenotypes in

\footnotetext{
5 In Hanahan and Weinberg (2000) they claim cancer is a population of cells with the following features (1) Self stimulation of growth (2) resist inhibitory signals (3) apoptosis avoidance (4) angiogenesis (5) unlimited replication (6) metastasis.
} 
populations to apply natural selection style models to cancer dynamics. This is not to say that there are not important differences. Cancer is a by-product of the adaptation and evolution of complex organisms. Thus, many (most) adaptations of complex tumors are themselves byproducts of our evolutionary history.

\section{Why are metazoans so susceptible to cancer?}

We have described cancer as process in which somatic cell evolve to have features of a Darwinian population. Cancer progression involves transitions in the levels of selection similar to the major transitions of evolution. Of course, major transitions take hundreds of thousands of years, but cancer progression from the first progenitor cells usually takes decades, though it can occur much more quickly. How is it that metazoans are particularly vulnerable to cancer? We have argued that this is in part due to cancer cells' cooption of organismic adaptations; here we claim additionally that cancer is a byproduct not only of organismic adaptations, but features distinctive to the metazoan clade (though, see, e.g., Aktipis et al. 2015 for a discussion of "cancer like" phenomena in plants).

The fact that many parts of our body (from organs, to cells, to organelles, to features of the genome themselves) are modular and thus quasi-independent, or can evolve or change over time without affecting the fitness of other parts, has made it possible for complex adaptations like the vertebrate limb to evolve. It also makes a particular type of defection possible. The very features that enable evolvability (quasi-independence, plasticity), also permit a tumor to arise or remain undetected. Thus, that cancer happens is in part a product of our being a highly evolvable lineage; in other words, it is a product of our fitness from a clade level perspective. The plasticity that enables metazoan cells to pass from epithelial to mesenchymal and then from mesenchymal to epithelial phenotypes, is what makes metastasis possible in carcinoma. While plants can suffer from uncontrolled cellular growths, the lack of an EMT, and thus cellular motility in plants prevents the possibility of metastatic tumors.

There is a history of selection for a number of traits that are recruited or deployed to promote tumor growth. Many traits that contribute to organismic fitness (or, fitness at the level of the whole organism) also are "coopted" by cancer cells (Crespi and Summers 2006). For instance, cellular plasticity, extensive proliferative capacity, mobility, and availability of lymphatic and vascular channels for cell migration all contribute to embryogenesis, wound healing, tissue renewal, and placental implantation. They also contribute to cancer initiation and progression. In other words, cancer is both product and byproduct of a multi-level selection process: the process of selection among cells within whole organisms and, a by-product of selection between individual organisms.

The way cancer uses local micro-environment can be understood in reference to the adaptations the normally functioning cell lineages have, in light of selection at the level of the organism as a whole. Metastatic cells acquire their mesenchymal phenotype because they both send and receive signals to surrounding tissue that permit the breakdown of the extracellular matrix, and re-activate the very same 
"protocols" involved in the EMT (epithelial-mesenchymal transition) in development and wound healing. EMT is the process by which epithelial cells lose their polarity and cell-cell adhesion allowing them to be mobile. Cancer also coopts the signaling networks it is a part of; and, it "re-ignites" the very same internal programs that make possible wound healing and development, over the course of its natural history. In other words, subversion in cancer is only made possible by taking advantage of those very mechanisms by which subversion is (generally speaking) prevented: those very same signaling routes that ordinarily foster or further cooperative activity.

The transitions cancer goes through as it goes metastatic mirror the major transitions of evolution. The same features that make multicellularity possible operate in cancer progression to metastasis: the development of shared fitness interests; genetic bottlenecks (with both stem cells, and metastasis); division of labor (variable fitness of cancer cells in different tissue microenvironments); export of fitness (to the tumor as a whole); and rarely, perhaps, functional integration. Just as the multilevel selection framework can help explain the major transitions it can also help explain the major transitions in cancer phenotype.

\section{Conclusions}

We have argued that cancer is a multi-level evolutionary process. The accumulation of mutations (and epigenetic changes) in neoplastic progression can be understood as an evolutionary process; both individual cells and lineages in a cancer may be more or less successful in progressing to metastasis, depending upon features like the timing and acquisition of particular mutation types, or chromosomal instability. Cancer also coopts signaling systems that evolved in service of higher levels of organization, systems that ordinarily regulate wound healing, development and immune response. At advanced stages, the combined tumor and stroma can be viewed as an integrated entity, i.e., a collective with organization and fitness as a whole (MLS2 as opposed to MLS1). Cancer is an exemplary case multi-level selection process: it evolves at the level of populations of cells, competition among cell lineages, via cooption or a kind of cross-level expatiation, and as a group.

\section{References}

Aktipis CA, Boddy AM, Jansen G, Hibner U, Hochberg ME, Maley CC, Wilkinson GS (2015) Cancer across the tree of life: cooperation and cheating in multicellularity. Phil Trans R Soc B 370(1673):20140219

Anderson A, Weaver A, Cummings PT, Quaranta V (2006) Tumor morphology and phenotypic evolution driven by selective pressure from the microenvironment. Cell 127(5):905-915

Bissell M, Hines WC (2011) Why don't we get more cancer? A proposed role of the microenvironment in restraining cancer progression. Nat Med 17(3):320-329

Cairns J (1975) Mutation selection and the natural history of cancer. Nature 255:197-200

Cairns J (1978) Cancer science and society. Freeman and Co, San Francisco

Crespi B, Summers K (2006) Evolutionary biology of cancer. Trends Ecol Evol 20(10):545-551 
Damuth J, Heisler IL (1988) Alternative formulations of multilevel selection. Biol Phil 3(4):407-430

Egeblad M, Nakasone ES, Werb Z (2010) "Tumors as organs: complex tissues that interface with the entire organism”. Dev Cell 18:884-901

Fisher R (1930) The genetical theory of natural selection. Clarendon, Oxford

Frank SA (2007) Dynamics of cancer: incidence, inheritance and evolution. Princeton, New Jersey

Frank SA, Nowak MA (2004) Problems of somatic mutation and cancer. BioEssays 26(3):291-299

Gatenby RA et al (2006) Acid-mediated tumor invasion: a multidisciplinary study. Cancer Res 66:5216-5223

Gatenby RA et al (2007) Cellular adaptations to hypoxia and acidosis during somatic evolution of breast cancer. Br J Cancer 97(5):646-653

Gause GF (1966) Aspects of antibiotic research. Chem Ind 36:1506-1513

Gavert N, Ben-Ze'ev A (2010) Coordinating changes in cell adhesion and phenotype during EMT-like processes in cancer. F1000 Biol Rep 8(2):86

Gerlinger M, Rowan AJ, Horswell S, Larkin J, Endesfelder D, Gronroos E, Swanton C (2012) Intratumor heterogeneity and branched evolution revealed by multiregion sequencing. New Eng $\mathrm{J}$ Med 366(10):883-892

Germain PL (2012) Cancer cells and adaptive explanations. Biol Phil 27(6):785-810

Godfrey-Smith P (2009) Darwinian populations and natural selection, vol 22. Oxford University Press, Oxford

Greaves M, Maley CC (2012) Clonal evolution in cancer. Nature 481(7381):306-313

Gupta PB, Chaffer CL, Weinberg RA (2009) Cancer stem cells: mirage or reality? Nat Med 15(9):1010-1012

Hanahan D, Weinberg RA (2000) The hallmarks of cancer. Cell 100(1):57-70

Hausman DM (2012) Health, naturalism, and functional efficiency. Phil Sci 79(4):519-541

Jordan CT, Guzman ML, Noble M (2006) Cancer stem cells. New Eng J Med 355(12):1253-1261

Karamysheva AF (2008) Mechanisms of angiogenesis. Biochemistry (Moscow) 73(7):751-762

Klein CA, Blankenstein TJ, Schmidt-Kittler O, Petronio M, Polzer B, Stoecklein NH, Riethmüller G (2002) Genetic heterogeneity of single disseminated tumour cells in minimal residual cancer. Lancet 360(9334):683-689

Komarova NL, Wodarz D (2004) The optimal rate of chromosome loss for the inactivation of tumor suppressor genes in cancer. Proc Natl Acad Sci USA 101(18):7017-7021

Komarova NL, Wodarz D (2005) Drug resistance in cancer: principles of emergence and prevention. Proc Natl Acad Sci 102(7):9714-9719

Lewontin RC (1970) The units of selection. Ann Rev Ecol Syst 1:1-18

Lloyd E (2012) Units and levels of selection. In: Edward NZ (ed) The stanford encyclopedia of philosophy. URL $=<$ http://plato.stanford.edu/archives/win2012/entries/selection-units/>

MacArthur RH, Wilson EO (1967) The theory of island biogeography. Princeton University Press, New Jersey

Martincorena I et al (2015) High burden and pervasive positive selection of somatic mutations in normal human skin. Science 348(6237):880-886

Merlo LMF, Pepper JW, Reid BJ, Maley CC (2006) Cancer as an evolutionary and ecological process. Nat Rev Cancer 6(12):924-935

Michod RE (1997) Evolution of the individual. Am Nat 150(S1):S5-S21

Michod RE, Herron MD (2006) Cooperation and conflict during evolutionary transitions in individuality. J Evol Biol 19:1406-1409

Michor F, Iwasa Y, Nowak MA (2004) Dynamics of cancer progression. Nat Rev Cancer 4(3):197-205

Morange M (2012) What history tells us XXVIII. What is really new in the current evolutionary theory of cancer? J Biosci 37(4):609-612

Mori $\mathrm{H}$ et al (2002) Chromosome translocations and covert leukemic clones are generated during normal fetal development. Proc Natl Acad Sci 99(12):8242-8247

Mueller M, Fuesnig NE (2004) "Friends or foes - bipolar effects of the tumor stroma in cancer. Nat Rev Cancer 4:839-849

Navin N, Kendall J, Troge J, Andrews P, Rodgers L, McIndoo J, Wigler M (2011) Tumour evolution inferred by single-cell sequencing. Nature 472(7341):90-94

Nguyen LV, Vanner R, Dirks P, Eaves CJ (2010) Cancer stem cells: an evolving concept. Nat Rev Cancer 12(2):220-228

Nowak MA (2006) Evolutionary dynamics: exploring the equations of life. Harvard University Press, Massachusetts 
Nowell PC (1976) The clonal evolution of tumor cell populations. Science 194:23-28

Odling-Smee FJ, Laland K, Feldman M (2003) Niche construction: a neglected process in evolution. Princeton University Press, New Jersey

Okasha S (2005) Multi-level selection and the major transitions in evolution. Phil Sci Proc 72:1013-1025

Okasha S (2006) Evolution and the levels of selection. Oxford University Press, Oxford

Olumi AF, Grossfeld GD, Hayward SW, Carroll PR, Tlsty TD, Cunha GR (1999) Carcinoma-associated fibroblasts direct tumor progression of initiated human prostatic epithelium. Cancer Res 59(19):5002-5011

Pepper JW, Sprouffske K, Maley CC (2007) Animal cell differentiation patterns suppress somatic evolution. PLoS Comput Biol 3(12):e250

Psaila B, Lyden D (2009) The metastatic niche: adapting the foreign soil. Nat Rev Cancer 9(4):285-293

Queller D, Strassman J (2009) Beyond society: the evolution of organismality. Phil Trans R Soc B 364:3143-3155

Redfield RJ (2002) Is quorum sensing a side effect of diffusion sensing? Trends Microbiol 10:365-370

Sakr WA et al (1993) The frequency of carcinoma and intraepithelial neoplasia of the prostate in young male patients. J Urol 150(2 Pt 1):379-385

Sober E, Wilson DS (1999) Unto others: the evolution and psychology of unselfish behaviour. Harvard University Press, Massachusetts

Sterelny KR, Joyce B, Calcott B, Fraser B (2014) Cooperation and its evolution. MIT Press, Massachusetts

Turner JR (1977) Butterfly mimicry: the genetical evolution of an adaptation. Evol Biol 10:163-206

Weigelt B, Glas AM, Wessels LF, Witteveen AT, Peterse JL, van't Veer LJ (2003) Gene expression profiles of primary breast tumors maintained in distant metastases. Proc Natl Acad Sci 100(26):15901-15905

Weinberg RA (2014) The biology of cancer, 2nd (edn.). Garland Science, NY

West S, Diggle SP, Buckling A, Gardner A, Griffin AS (2007) The social lives of microbes. Ann Rev Ecol Evol Syst 38:53-77

Williams P, Winzer K, Chan W, Ćamara M (2007) Look who's talking: communication and quorum sensing in the bacterial world. Philos Trans R Soc Lond, Ser B 362(1483):1119-1134

Wilson DS (1975) A theory of group selection. Proc Natl Acad Sci 72(1):143-146

Wodarz D, Komarova NL (2014) Dynamics of cancer: mathematical foundations of oncology. World Scientific Publishing Co, Singapore

Yachida S et al (2010) Distant metastasis occurs late during the genetic evolution of pancreatic cancer. Nature 467:1114 